\title{
Nucleotide variations of TP53 exon 4 found in intracranial meningioma and in silico prediction of their significance
}

\author{
ANJA BUKOVAC ${ }^{1,2}$, ANJA KAFKA ${ }^{1,2}$, RENO HRAŠĆAN ${ }^{3}$, TOMISLAV VLADUŠIĆ ${ }^{3}$ and NIVES PEĆINA-ŠLAUS ${ }^{1,2}$ \\ ${ }^{1}$ Laboratory of Neurooncology, Croatian Institute for Brain Research; ${ }^{2}$ Department of Biology, School of Medicine, \\ University of Zagreb; ${ }^{3}$ Department of Biochemical Engineering, Faculty of Food Technology and Biotechnology, \\ University of Zagreb, 10000 Zagreb, Croatia
}

Received April 9, 2019; Accepted August 20, 2019

DOI: $10.3892 / \mathrm{mco} .2019 .1936$

\begin{abstract}
The aim of the present study was to identify TP53 exon 4 mutations in patients with meningioma and to investigate their potential association with specific tumor pathology. Nucleotide alterations were investigated in 48 meningiomas via the direct sequencing of TP53 exon 4 in patient tumor and blood samples using the DNA Sanger method with the BigDyeTerminator v3.1 Cycle Sequencing kit and Applied Biosystems 3730XL apparatus. The results revealed that TP53 exon 4 was frequently altered in meningioma, occurring in $60.4 \%$ of the patients investigated. A total of 18 different alterations were detected in the meningioma samples assessed in the current study. The majority of these appeared more than once and some were repeatedly identified in several patients. Changes at codons 72 (c.215G >C) and 62 (c.186delA) were highly prevalent, occurring in $44.8 \%$ of patients. Other changes detected via frequency analysis included: Five substitutions on codon 105 (c.315C >T); four insertions on codon 70 (c.209_210insG); three insertions on codon 64 (c.190C>G), 82 (245C>T; 245delC; 243_244insA) and 104 (c.312G>A); and two insertions on codons 108 (c.322G>C), 71 (c.213C $>A)$, 73 (c.217G >A), 91 (c.271T>C) and 100 (c.300G>T). Codons 68 (c.202_203insT), 77 (c.229C>T), 88 (c.263C>G) and 92 (c.276C $>$ A) were altered once. Alterations on codons 82, 91, 108, 104, 105, 70 and 92 were characterized as possibly damaging by PolyPhen-2 and Mutation Taster 2 tools. The current study also demonstrated that nucleotide alterations were significantly associated with the loss of $\mathrm{p} 53$ expression $(\mathrm{P}=0.04)$ and female patients $(\mathrm{P}=0.049)$, particularly codon 72 . The results present novel data on the mutational spectrum of TP53 in meningeal brain tumors.
\end{abstract}

Correspondence to: Professor Nives Pećina-Šlaus, Laboratory of Neurooncology, Croatian Institute for Brain Research, School of Medicine, University of Zagreb, Šalata 12/3, 10000 Zagreb, Croatia E-mail: nina@mef.hr

Key words: TP53, exon 4, meningioma, mutations, DNA variants, Sanger sequencing

\section{Introduction}

A multitude of aberrant signaling pathways have been implicated in meningioma tumorigenesis. Potentially responsible signaling pathways include RB/p53, MAPK, PI3K/Akt, Wnt, and many others (1). Many genes involved in cell-cycle control, cell growth and proliferation have been reported to be mutated or aberrantly expressed and often associated with high grade meningioma $(2,3)$. However, the molecular etiology and pathogenesis of this tumor still seek many answers. The p53 pathway is involved in and often dysregulated in anaplastic meningiomas. The involvement of p53 pathway in meningiomas was first observed by marked loss of expression of p53 protein (4). Tumor suppressor p53, encoded by the TP53 gene, is a key protein involved in many major cellular tumor suppression processes such as the control of cell cycle and apoptosis. In the present study we wanted to investigate presence of mutations of TP53's exon 4 relevant for intracranial meningioma. The exons 2-4 encode for the two transactivation domains of $\mathrm{p} 53$ protein (amino acids 1 through 42 and 43-62) (5). Moreover, exons 4-8 code for the so called core domain of p53, an important domain by which the protein binds to DNA.

In spite of the fact that tumor suppressor gene TP53 is the most frequently mutated gene in human cancers overall, the findings on TP53 in meningioma are scarce and controversial. In order to understand the role of p53 in the etiology and pathogenesis of meningioma thorough investigation is still necessary (6). Past studies have examined alterations in TP53 tumor suppressor gene in meningiomas and it is generally considered that mutations of TP53 are not frequent and thus not important for meningioma biology (7-11). However, this is a misconception, because when examining literary reports in more detail it becomes obvious that only several meningioma cases were reported and few exons sequenced. Opposing studies relevant to our work provide findings on p53 pathway in meningioma development (12). For example, the correlation of p53 protein expression with histological tumor grade and meningioma recurrence $(13,14)$ and the loss of detectable MDM2 protein in high grade meningiomas $(15,16)$ are all in favor of p53's involvement. Recently, a meningioma candidate tumor suppressor gene called maternally expressed gene 3 (MEG3) has been identified to transactivate TP53 (17). Loss of MEG3 expression and its allelic loss were associated with 
higher meningioma grades. MEG3, a noncoding RNA with antiproliferative functions, is strongly expressed in normal arachnoidal cells, but absent in meningioma cell lines.

Cancer genome sequencing of 12 different tumor types has shown that $42 \%$ of investigated cases carry mutant TP53 genes (18). Yet, different tumors displayed quite different frequencies of TP53 mutations, some harboring only $2.2 \%$, while others even $95 \%$. It is well known that p53 has many functions. It acts as a transcription factor activated upon sensing cellular stress of different kinds. Its accumulation affects the cell cycle, DNA repair, apoptosis, senescence and cell differentiation. In normal cells p53 protein levels are kept low by the following mechanism: p53 upregulates its negative regulator, E3 ubiquitin-protein ligase MDM2 which causes rapid ubiquitination and degradation of p53 by the proteasome $(18,19)$.

Meningiomas account for approximately $30 \%$ of primary intracranial and intraspinal neoplasms. It is believed nowadays that the tumor arises from the arachnoidal cap cells of the leptomeninges. Most meningiomas are characterized as benign, slowly growing tumors with long survival time and are classified as WHO grade I $(20,21)$. However, meningiomas can show an aggressive nature. In between benign and malignant types are atypical meningiomas characterized by increased mitotic activity, brain invasion and a higher risk for recurrence. They are WHO grade II tumors and represent about $5-7 \%$ of all meningioma cases. Grade III tumors-anaplastic meningiomas, are typically associated with brain invasion and recurrence. They exhibit true malignancy and represent 3\% of all meningioma cases with the low overall 10 -year survival rate of $14.2 \%(22,23)$. Meningiomas as a whole can display a broad spectrum of clinical, histological and cytogenetic features. Hence, 15 different subtypes are described in the current pathohistological classification of meningiomas. The considerable variability in the biological behavior could be observed even within the same WHO grade. So, histologically distinct subtypes of benign meningiomas may exhibit high risk of recurrence, or even evolve into atypical and anaplastic subtypes (24-26). Our understanding of the genetic profiles of sporadic meningiomas only recently started to uncover owing to large-scale genomic analyses (27-29). However, relevant genetic events for atypical and anaplastic cases as well as molecular mechanisms of meningioma progression still need to be fully understood. It has been well documented that the neurofibromatosis type 2 gene (NF2) located on 22q is inactivated through mutation and loss of heterozygosity ( $\mathrm{LOH})$ in the majority of meningiomas (30). Consequent loss of NF2 encoded protein merlin, is a consistent finding in all neurofibromatosis type 2 associated meningiomas and in about half of sporadic benign cases (31).

Our investigation that focused on exon 4 , that codes for the functionally important domains for p53 protein, demonstrated that mutations that impact the p53's transactivation and DNA binding domains are present in our group of collected meningiomas.

\section{Patients and methods}

Tissue samples. We conducted our study by collecting 48 paired meningioma tumors and autologous blood from the Departments of Neurosurgery and Pathology, Hospital Center 'Sisters of Charity', Zagreb, Croatia, following patients' consents. Collected tumor tissues were frozen in liquid nitrogen and were kept at $-80^{\circ} \mathrm{C}$. The peripheral blood samples were collected in EDTA and processed immediately. Using the magnetic resonance imaging (MRI) tumor lesions were diagnosed in different cerebral regions, with the surrounding zone of perifocal edema. During the operative procedure the tumor was maximally reduced using a microneurosurgical technique. No family history of brain tumors was recorded in the collected patients and they did not undergo chemotherapy or radiotherapy prior to surgery. All meningioma samples were first evaluated after resection and then studied by neuropathologists who classified them according to the WHO criteria (21). There were 17 meningothelial meningiomas, 7 fibrous (fibroblastic), 15 transitional (mixed), 1 psammomatous, 4 angiomatous, 2 atypical and 2 anaplastic. Clinical and pathological data are shown in Table I. Twenty-six patients were females and 22 males. The age of patients varied from 32 to 79 (mean age $=63.19$, median 66 years). The mean age at diagnosis for females was 65.84 , and for males 60.05 years. All procedures involving human participants were performed in accordance with the ethical standards of the Institutional and National Research Committee, and with the Declaration of Helsinki. Ethical approval was obtained from the Ethical Committee of School of Medicine University of Zagreb (approval no. 380-59-10106-14-55/147; Class: 641-01/14-02/01,1. 07. 2014) and of the University Hospital Center 'Sisters of Mercy' (approval no. EP-7426/14-9,11. 06. 2014). Verbal informed consent was obtained from all patients included in the present study.

DNA extraction. We obtained tumor DNA by homogenizing approximately $0.5 \mathrm{~g}$ of each specimen tumor tissue with $1 \mathrm{ml}$ extraction buffer (10 mM Tris $\mathrm{HCl}$; $0.1 \mathrm{M}$ EDTA; $0.5 \%$ sodium dodecyl sulfate, $\mathrm{pH}$ 8.0) and incubated with proteinase $\mathrm{K}$ (100 mg/ml; Sigma; overnight at $37^{\circ} \mathrm{C}$ ). Phenol/chloroform extraction and ethanol precipitation followed. Leukocyte DNA was extracted from the blood samples. Three $\mathrm{ml}$ of blood was lysed with $7 \mathrm{ml}$ distilled water and centrifuged ( $15 \mathrm{~min} / 5.000 \mathrm{~g})$. After that, DNA extraction was the same as for the tissue samples. The concentration of double-stranded DNA from the tumors and blood was quantified with Nanodrop.

Polymerase chain reaction, DNA sequencing reactions. We investigated mutational status of exon 4 of the TP53 gene in our collected meningioma sample by direct Sanger sequencing of this exon. Each PCR reaction was performed in a total reaction volume of $25 \mu \mathrm{l}$ with the following final optimized concentrations: $0.2 \mathrm{mM}$ of each dNTP, $3 \mathrm{mM} \mathrm{MgCl}_{2}, 0.2 \mu \mathrm{M}$ of each primer (Sigma-Aldrich), 1x Flexi buffer and $0.5 \mathrm{U}$ of GoTaq ${ }^{\circledR}$ G2 Hot Start Polymerase (both Promega). Reaction conditions were as follows: $96^{\circ} \mathrm{C} / 5 \mathrm{~min}$ (one cycle), 45 cycles of $96^{\circ} \mathrm{C} / 30 \mathrm{sec}, 51^{\circ} \mathrm{C} / 45 \mathrm{sec}, 72^{\circ} \mathrm{C} / 30+1 \mathrm{sec}$, and the final step of $72^{\circ} \mathrm{C} / 10 \mathrm{~min}$. Primers used for the TP53's exon 4 were $5^{\prime}$-GAT GCTGTCCGCGGACGATAT-3', 5'-CGTGCAAGTCACAGA CTTGGC-3'. The PCR products of the target sequence were 247-bp long and resolved on $2 \%$ agarose gels.

Nucleotide variations were discovered by Sanger sequencing using the Big DyeTerminator v3.1 Cycle Sequencing kit. An 
Table I. Nucleotide alterations of TP53 exon 4 and the pathohistological type, grade and demographic variables of the collected meningioma samples.

\begin{tabular}{|c|c|c|c|c|}
\hline Patient & TP53 e4 nucleotide alterations & Meningioma type & Age (years) & Sex \\
\hline 1 & c. $215 \mathrm{G}>\mathrm{C}$, p.R72P, Mi; c.186delA, p.E62del, Fr & Meningothelial (I) & 72 & M \\
\hline 2 & c.186delA, p.E62del, Fr; c.209_210G, p.A70ins, Fr & Meningothelial (I) & 70 & $\mathrm{~F}$ \\
\hline 3 & No mutation & Meningothelial (I) & 62 & $\mathrm{~F}$ \\
\hline 4 & No mutation & Meningothelial (I) & 64 & M \\
\hline 5 & c.190C >G, p.P64A, Mi; c.215G>C, p.R72P, Mi & Meningothelial (I) & 65 & M \\
\hline 6 & No mutation & Meningothelial (I) & 35 & M \\
\hline 7 & c.186delA, p.E62del, Fr & Meningothelial (I) & 55 & $\mathrm{~F}$ \\
\hline 8 & c. $213 \mathrm{C}>\mathrm{A}, \mathrm{p} . \mathrm{P} 71 \mathrm{P}, \mathrm{S}$ & Meningothelial (I) & 47 & $\mathrm{~F}$ \\
\hline 9 & No mutation & Meningothelial (I) & 70 & M \\
\hline 10 & No mutation & Meningothelial (I) & 54 & M \\
\hline 11 & c.186delA, p.E62del, Fr; c.215G>C, p.R72P, Mi; c.276C>A, p.P92P, S & Meningothelial (I) & 62 & $\mathrm{~F}$ \\
\hline 12 & $\begin{array}{l}\text { c. } 217 \mathrm{G}>\mathrm{A}, \mathrm{p} . \mathrm{V} 73 \mathrm{M}, \mathrm{Mi} ; \text { c.300G }>\mathrm{T}, \mathrm{p} . \mathrm{Q} 100 \mathrm{H}, \mathrm{Mi} \\
\text { c.312G }>\text { A, p.Q104Q, S; c.315C>T, p.G105G, S }\end{array}$ & Meningothelial (I) & 71 & M \\
\hline 13 & $\begin{array}{l}\text { c.215G >C, p.R72P, Mi; c.229C>T, p.P77S, Mi; c.245C>T, p.P82L, Mi; } \\
\text { c.263C>G, p.A88G, Mi; c.271T>C, p.W91R, Mi }\end{array}$ & Meningothelial (I) & 40 & M \\
\hline 14 & $\begin{array}{l}\text { c.186delA, p.E62del, Fr; c.215G>C, p.R72P, Mi; c.271T>C, p.W91R, Mi; } \\
\text { c.312G>A, p.Q104Q, S }\end{array}$ & Meningothelial (I) & 63 & $\mathrm{~F}$ \\
\hline 15 & c.215G>C, p.R72P, Mi; c.322G>C, p.G108R, Mi & Meningothelial (I) & 62 & M \\
\hline 16 & No mutation & Meningothelial (I) & 75 & $\mathrm{~F}$ \\
\hline 17 & c.186delA, p.E62del, Fr; c.209_210G, p.A70ins, Fr & Meningothelial (I) & 67 & $\mathrm{~F}$ \\
\hline 18 & No mutation & Fibrous (I) & 54 & M \\
\hline 19 & c.186delA, p.E62del, Fr; c.190C>G, p.P64A, Mi; c.209_210G, p.A70ins, Fr & Fibrous (I) & 63 & $\mathrm{~F}$ \\
\hline 20 & No mutation & Fibrous (I) & 45 & M \\
\hline 21 & No mutation & Fibrous (I) & 51 & $\mathrm{~F}$ \\
\hline 22 & No mutation & Fibrous (I) & 73 & $\mathrm{~F}$ \\
\hline 23 & No mutation & Fibrous (I) & 66 & $\mathrm{~F}$ \\
\hline 24 & c. $215 \mathrm{G}>\mathrm{C}, \mathrm{p} . \mathrm{R} 72 \mathrm{P}, \mathrm{Mi}$ & Fibrous (I) & 74 & $\mathrm{~F}$ \\
\hline 25 & No mutation & Transitional (I) & 56 & $\mathrm{~F}$ \\
\hline 26 & No mutation & Transitional (I) & 61 & M \\
\hline 27 & c.243_244insA, p.P82ins, Fr; c.300G>T, p.Q100H, Mi & Transitional (I) & 45 & M \\
\hline 28 & No mutation & Transitional (I) & 72 & $\mathrm{~F}$ \\
\hline 29 & c.186delA, p.E62del, Fr; c.315C>T, p.G105G, S & Transitional (I) & 74 & $\mathrm{~F}$ \\
\hline 30 & c. $215 \mathrm{G}>\mathrm{C}, \mathrm{p} . \mathrm{R} 72 \mathrm{P}, \mathrm{Mi}$ & Transitional (I) & 75 & M \\
\hline 31 & c.215G>C, p.R72P, Mi & Transitional (I) & 32 & M \\
\hline 32 & c.202_203insT,p.E68ins, Fr & Transitional (I) & 77 & M \\
\hline 33 & c.186delA, p.E62del, Fr & Transitional (I) & 71 & $\mathrm{~F}$ \\
\hline 34 & c.186delA, p.E62del, Fr; c.315C>T, p.G105G, S & Transitional (I) & 64 & $\mathrm{~F}$ \\
\hline 35 & $\begin{array}{l}\text { c. } 215 \mathrm{G}>\mathrm{C}, \text { p.R72P, Mi c. } 217 \mathrm{G}>\mathrm{A}, \mathrm{p} . \mathrm{V} 73 \mathrm{M}, \mathrm{Mi} \text {; c.312G>A, p.Q104Q, S; } \\
\text { c. } 315 \mathrm{C}>\mathrm{T} \text {, p.G105G, S }\end{array}$ & Transitional (I) & 66 & $\mathrm{~F}$ \\
\hline 36 & c.186delA, p.E62del, Fr & Transitional (I) & 73 & $\mathrm{~F}$ \\
\hline 37 & c.186delA, p.E62del, Fr; c.215G>C, p.R72P, Mi & Transitional (I) & 67 & $\mathrm{~F}$ \\
\hline 38 & c.186delA, p.E62del, Fr & Transitional (I) & 79 & $\mathrm{~F}$ \\
\hline 39 & c. $315 \mathrm{C}>\mathrm{T}, \mathrm{p} . \mathrm{G} 105 \mathrm{G}, \mathrm{S} ;$ c.322G>C, p.G108R, Mi & Transitional (I) & 61 & $\mathrm{~F}$ \\
\hline 40 & c.190C>G, p.P64A, Mi; c.215G>C, p.R72P, Mi & Psammomatous (I) & 60 & $\mathrm{~F}$ \\
\hline 41 & No mutation & Angiomatous (I) & 66 & M \\
\hline 42 & No mutation & Angiomatous (I) & 39 & M \\
\hline 43 & c. $215 \mathrm{G}>\mathrm{C}, \mathrm{p} . \mathrm{R} 72 \mathrm{P}, \mathrm{Mi}$ & Angiomatous (I) & 70 & $\mathrm{~F}$ \\
\hline 44 & c.209_210insG, p.A70ins, Fr & Angiomatous (I) & 78 & M \\
\hline 45 & No mutation & Atypical (II) & 76 & M \\
\hline 46 & No mutation & Atypical (II) & 73 & M \\
\hline 47 & c.213C>A, p.P71P, S; c.245delC, p.P82del, Fr & Anaplastic (III) & 71 & $\mathrm{~F}$ \\
\hline 48 & No mutation & Anaplastic (III) & 67 & M \\
\hline
\end{tabular}

TP53, tumor protein 53; e4, exon 4; I, grade I; II, grade II; III, grade III (malignant); Mi, missense; S, silent; Fr, frameshift; M, male; F, female. 
enzymatic clean-up using ExoI and FastAP from ThermoFisher Scientific was performed prior to sequencing. The BigDye Terminator 3.1 Sequencing Buffer for the set-up of the reactions was used and the plate was purified via a Sephadex plate after PCR. DNA sequencing reactions were analyzed on the Applied Biosystems 3730XL (Applied Biosystems) with 96 capillaries (Source BioScience $\mathrm{GmbH}$ ). All samples were sequenced twice with independent PCR reactions. Sequence analysis was carried out using NCBI Nucleotide Blast (https://blast.ncbi. nlm.nih.gov/Blast.cgi?PAGE_TYPE=BlastSearch). Reference sequence was used from GeneBank accession number U94788.1. The evaluation of pathogenicity was performed with the PolyPhen-2 (http://genetics.bwh.harvard.edu/pph2/) and ClinVar (https://www.ncbi.nlm.nih.gov/clinvar/) tools (32) and the prediction whether an amino acid substitution affects protein function with SIFT (Sorting Intolerant from Tolerant algorithm http://sift.jcvi.org/) and Mutation Taster2 (http://www.mutationtaster.org/) tools $(33,34)$.

Immunohistochemistry. Forty-six meningiomas were available for this analysis. The sections were immunostained using streptavidin-horseradish peroxidase/DAB (EnVisionTM, Dako REALTM) as previously described (2). The primary antibodies used for p53 detection were mouse anti-human monoclonal antibodies (clone DO-7; DAKO; diluted 1:25).

Statistical Analysis. All statistical evaluations were carried out using SPSS statistical package version 14.0 (SPSS Inc.). Following variables were tested for all patients: Meningioma histological subtype and malignancy grade, p53 mutational type, protein presence, sex and age. The normality of data was tested using the Shapiro-Wilk test. Pearson Chi-Square and Spearman's correlation were employed to test the relationships between the various parameters. $\mathrm{P}<0.05$ was considered to indicate a statistically significant difference.

\section{Results}

The frequency and type of mutations. Mutations of exon 4 of TP53 gene were very frequent and altogether detected in 29 meningioma patients $(60.42 \%)$. Several patients harbored more than one mutation. There were altogether 18 different mutations across our meningioma sample. Meningiomas with nucleotide alterations are listed in Table I. Sequencing results are shown in Fig. 1. Several changes have been characterized as polymorphisms in the literature and databases. Interestingly 13 observed alterations were appearing more than once, and some were repeatedly found in several meningioma patients. For example, out of 29 samples showing nucleotide alterations, 13 meningiomas had changes in codon 72 (44.8\%) when compared to blood DNA and database sequences (IARC T53 Database http://p53.iarc.fr/TP53GermlineMutations. aspx), making this change highly represented in meningioma. This frequent change is a well-known polymorphism CGC to $\mathrm{CCC}$, that was recently revised and categorized as a missense, resulting in amino acid change of ARG to PRO frequently found in different tumor types (c.215G>C; p.R72P; g.7676154C >G, Variant ID: 0000487638). The mutational frequency distributed across codons, their consequences and distribution to meningioma subtypes, are illustrated in Fig. 2.
Another highly represented mutation was at codon 62 (c.186delA; p.E62del; Variant ID: 0000487618, g.7579501del) where meningiomas showed deletion of nucleotide A when compared to normal sequence reported in databases (GAA coding for Glu). This mutation was also represented in $44.8 \%$ of total number of mutations found introducing a potential frameshift in meningioma patients. Other nucleotide substitutions that we identified and listed by the observed frequency included: Five substitutions on codon 105 (GGC to GGT; c.315C>T; p.G105G; g.7676054G>A, Variant ID: 0000487634) making this a silent mutation; four insertions on codon 70 (G was inserted in tumor sequence; c.209_210insG; p.A70ins; g.7676159_7676160ins1, Variant ID: 0000497667) introducing frameshift; three missense on codon 64 (CCC to GCC; c.190C>G; p.P64A; g.7676179G>C; Variant ID: 0000487614); three changes on codon 82 (one missense (245C>T; p.P82L, g.7676124G>A; Variant ID: 0000487640), one deletion (245delC; p.P82del, g.7676124del1; Variant ID: 0000497669) and one insertion (243_244insA; p.P82ins; g.7579444dup; Variant ID: 0000489041)); three silent on codon 104 (c.312G>A; p.Q104Q; g.7676057C>T, Variant ID: 0000487633), two missense on codon 108 (GGT to CGT; c.322G>C; p.G108R; g.7676047C>G, Variant ID: 0000487662), two silent on codon 71 (CCC to CCA, c.213C>A; p.P71P; g.7676156G>T, Variant ID: 0000487619), two missense on codon 73 (GTG to ATG, c.217G>A; p.V73M; g.7676152C>T, Variant ID: 0000487629), codon 91 (TGG to CGG, c.271T>C; p.W91R; g.7676098A>G, Variant ID: 0000487646) and codon 100 (CAG to CAT, c.300G>T; p.Q100H; g.7676069C>A, Variant ID: 0000487631). Finally, codons 68 (insertion of $\mathrm{T}$ nucleotide, GAG to GTA; c.202_203insT; p.E68ins, frameshift, Variant ID: 0000489055), 77 (CCA to TCA, c.229C>T; p.P77S, missense; g.7676140G>A, Variant ID: 0000487639), 88 (GCC to GGC; c. $263 \mathrm{C}>\mathrm{G}$; p.A88G, missense; g.7676106G>C, Variant ID: 0000487644) and 92 (TGG to CGG, c.276C>A; p.P92P, silent; g.7676093G >T, Variant ID: 0000487622) were changed only once (Fig. 2A). We submitted our variants to LOVD (Leiden Open Variation Database) and can be viewed at the following URL: https://databases.lovd.nl/shared/users/03344. All nucleotide alterations that we found were already listed in the TP53 mutation database (http://p53.iarc.fr/TP53GeneVariations. aspx) but not in connection to meningioma except for codon 72 .

There were altogether $27 / 58(46.6 \%)$ nucleotide alterations representing missense mutations, 20/58 (34.5\%) frameshift mutations and 11/58 (19\%) silent mutations (Fig. 2B). We defined frameshifts as truncating mutations, that may lead to non-functional p53, based on previous studies (35-37).

Immunohistochemistry brought additional data on the influence of TP53 mutational status to protein levels. There were altogether $47.8 \%$ of samples with p53 positive immunostain (Fig. 3A and B). However, when the positivity was distributed we have found that the group of samples harboring mutations expressed significantly less protein $(\mathrm{P}=0.04$; Fig. 2D). We further inspected if mutations of specific codons influenced p53 positivity and found that codon 105 was significantly associated to the lack of p53 $(\mathrm{P}=0.031)$ while codon 62 showed the same trend $(\mathrm{P}=0.065)$. Finally, we observed that $63 \%$ of meningiomas harboring missense and frameshift mutations did not express p53 protein. 
A Tumor c.215G>C; p.R72P
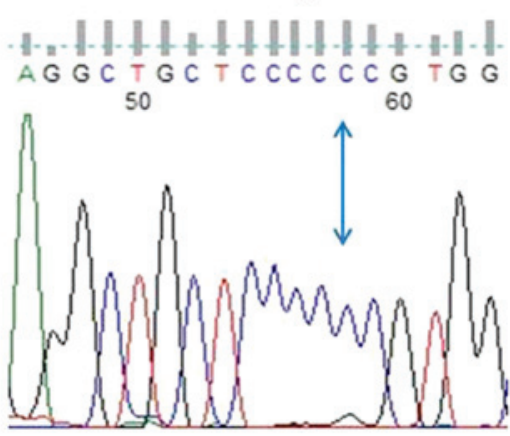

C Tumor c.209_210insG; p.A70ins

$G$ A G G C T G C GT C C C C G C G T

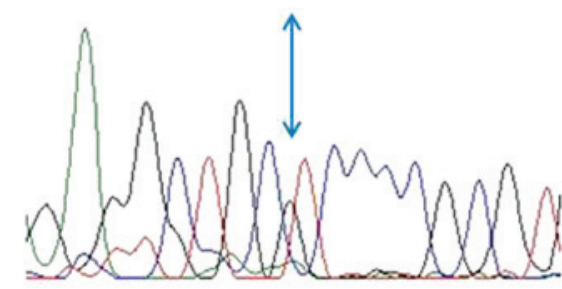

E Tumor c.322G>C;p.G108R

\section{B Wild type}

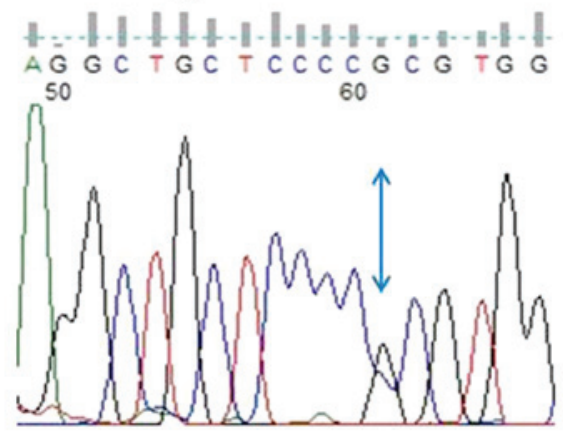

\section{Wild type}

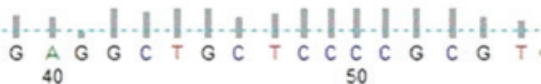

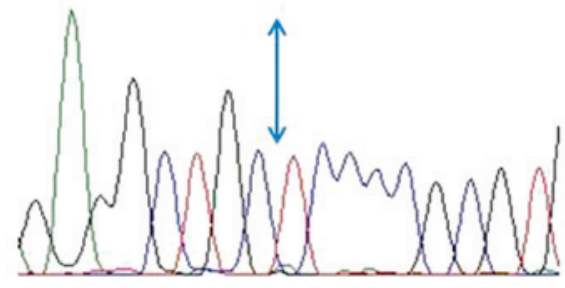

F Wild type

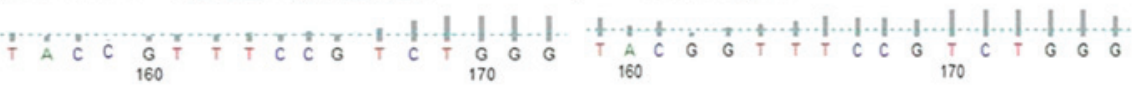

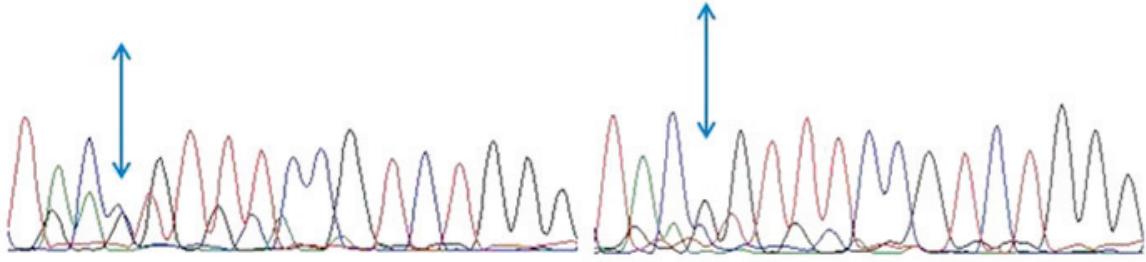

Figure 1. Representative DNA sequence of TP53 exon 4 nucleotide alterations in meningiomas. (A) Codon 72 tumor DNA (c.215G>C; p.R72P) and (B) codon 72 wild type data. (C) codon 70 tumor DNA (c.209_210insG; p.A70ins) and (D) codon 70 wild type data. (E) Codon 108 tumor DNA (c.322G>C; p.G108R) and $(\mathrm{F})$ wild type data. The blue arrows represent the locations of genetic alterations.

Association to histopathology, sex and age. We also wanted to test if the specific type of nucleotide alterations were associated to a specific meningioma histopathology or WHO grades. The results of statistical analysis obtained on our total sample demonstrate that significant differences in mutations were not associated to any specific meningioma subtype or grade $($ Chi-Square $=1.022 ; \mathrm{P}=0.336)$. Although meningothelial and transitional subtypes showed the highest numbers of both, codon 72 and 62 changes, and transitional subtype harbored the highest number of codon 105 changes, these values were not statistically significant (Fig. 2C).

Furthermore, possible associations to sex or age patterns were also inspected. The difference in overall mutational frequencies was statistically significantly associated to females. Women exhibited significantly higher frequency of alterations when compared to males (Chi square $=3.802$; sig=0.049). When analyzing the most frequently affected codons separately, it has been shown that females had significantly more alterations of codon 62 than male patients (Chi square=10.447; sig=0.001). We further compiled two large age categories, one consisting of individuals 63 years or younger $(n=20)$, and the other of individuals older than 63 years $(n=28)$, but observed that nucleotide alterations were not statistically associated to any specific age group.

In silico prediction of mutational consequences. In order to be able to explain the possible consequences of the observed mutations we performed the evaluation of pathogenicity with the PolyPhen-2 and ClinVar and the prediction if amino acid substitutions affect protein function with SIFT and Mutation Taster2 softwares $(33,38)$. The obtained results were similar between the databases with few exceptions. The majority of alterations were not validated $(87.5 \%)$. Transcriptional activity was classified as partially functional in $15.5 \%$ and functional in $8.6 \%$ of total mutations observed. There were $44.4 \%$ of alterations classified as benign and also $44.4 \%$ as deleterious and possibly damaging while $11.2 \%$ were undetermined. 

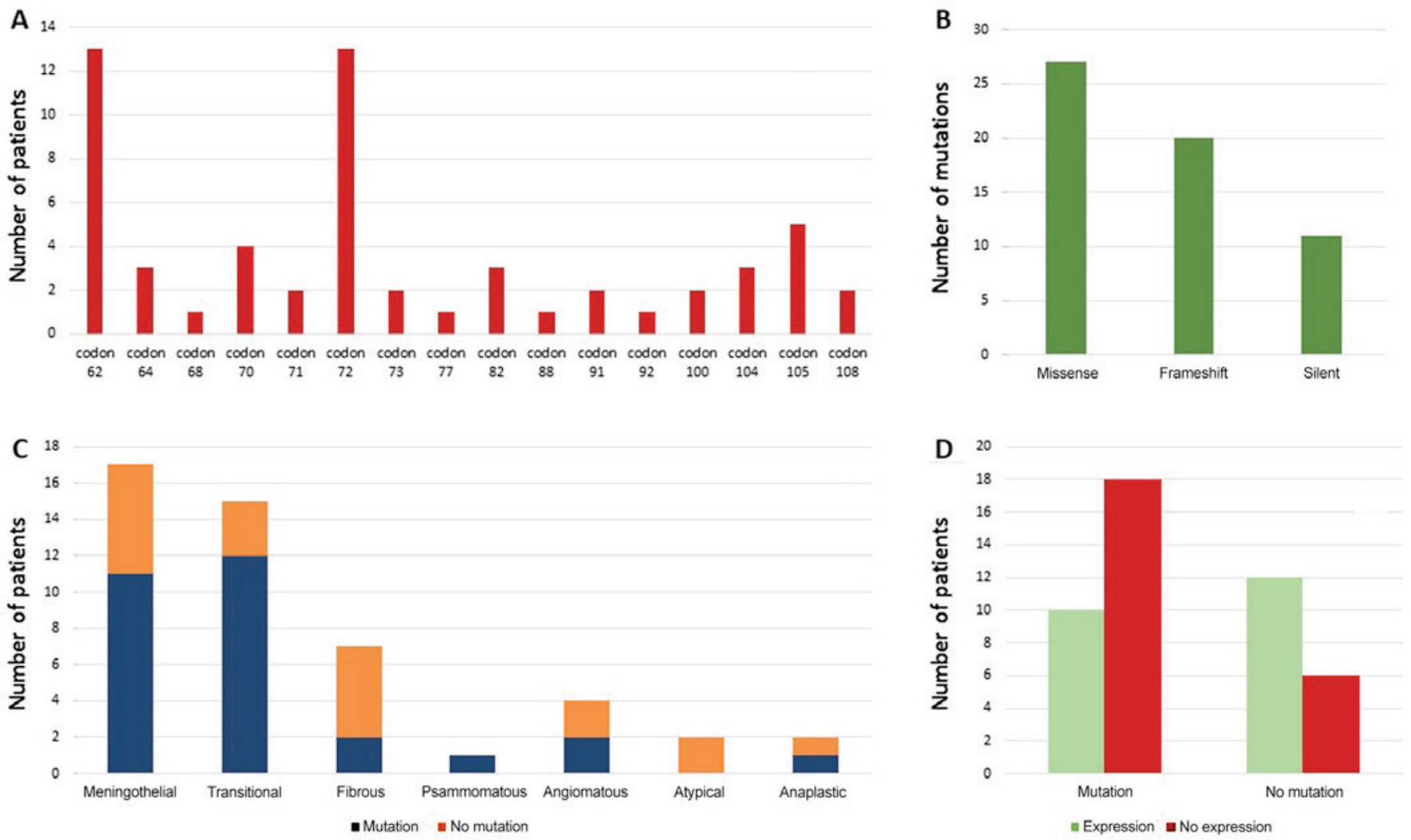

Figure 2. Summary of mutational frequencies and consequences across patients with meningioma. (A) Frequency of codon alterations. (B) The number of mutational consequence types. (C) The number of mutations distributed across different meningioma subtypes. (D) Number of patients with and without mutations expressing the p53 protein.
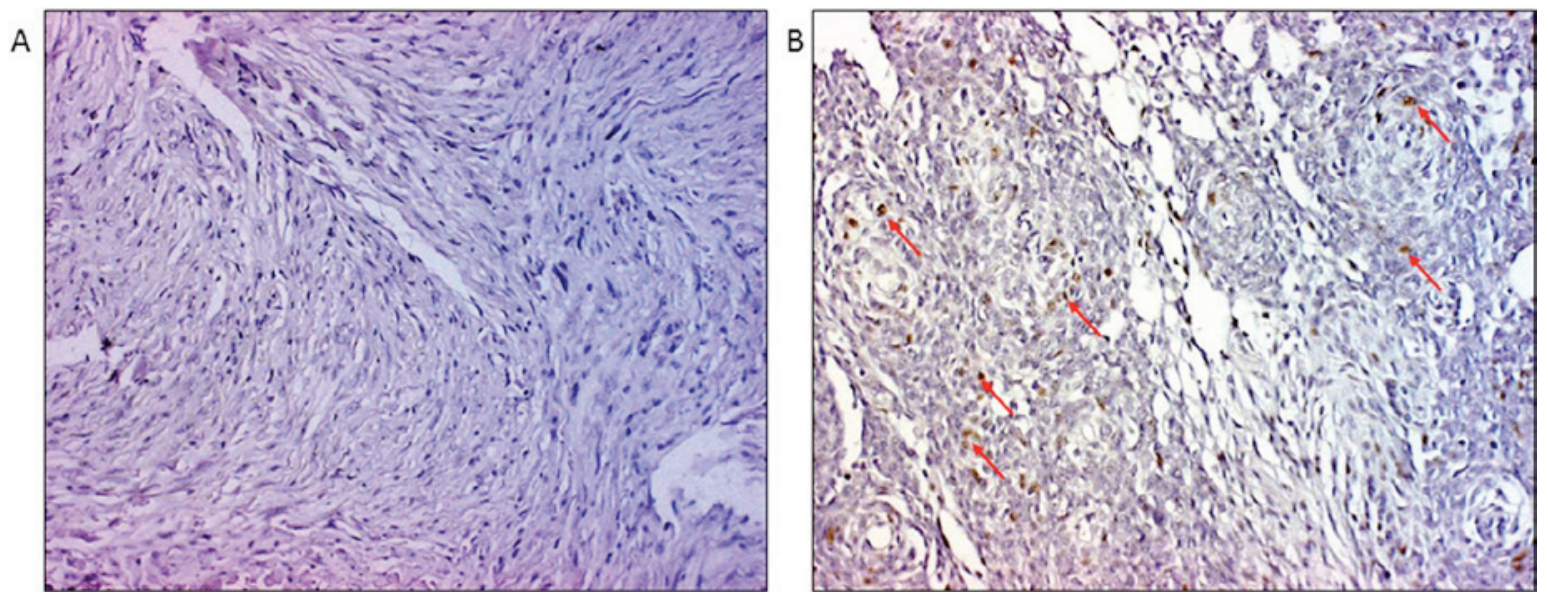

Figure 3. Immunohistochemistry of p53 expression in representative meningiomas. Tumors (A) without p53 expression and (B) with p53 expression. Red arrows indicate nuclear localization.

The alteration at codon 72 (c.215G>C; p.R72P) was validated and characterized by PolyPhen-2 and Mutation Taster2 as benign, but IARC p53 database characterizes this change as a missense and potentially disease causing. Alterations at codons 62 (c.186delA; p.E62del) and 71 (c.213C >A; p.P71P) were nonvalidated and with unknown functional consequences. The changes at codons 64 (c.190C $>$ G; p.P64A), 100 (c.300G $>$ T; p.Q100H), 77 (c.229C >T; p.P77S); 88 (c.263C >G; p.A88G); 73 (c.217G >A; p.V73M) and 68 (c.202_203insT; p.E68ins) were all nonvalidated, transcriptionally functional and benign. Codon 108 (c.322G>C; p.G108R) harbored nonvalidated, transcriptionally partially functional, but deleterious disease causing alteration found by SIFT, PolyPhen-2 and Mutation Taster2, while codon 91 (c.271T>C; p.W91R) harbored possibly damaging alteration found by both PolyPhen-2 and Mutation Taster2. Mutation Taster2 furthermore characterized mutations on codons 104 (c312G $>\mathrm{A}) ; 105$ (c.315C $>\mathrm{T}$; p.G105G); 70 (c.209_210insG; p.A70ins) and 92 (c.276C >A; p.P92P) as possibly disease causing. Of the three changes on codon 82 , the missense $(245 \mathrm{C}>\mathrm{T}$; p.P82L) was nonvalidated, transcriptionally functional and benign, while the deletion (245delC; p.P82del) was predicted as disease causing by 
Mutation Taster2 and insertion (243_244insA; p.P82ins) was characterized as possibly damaging by PolyPhen-2.

\section{Discussion}

Loss of p53 suppressor function is a common event in the development of a wide variety of human tumors and contributes to an increase in genetic instability and metastatic potential. It is implicated in many types of cancer and its mutations are present in substantial number of all sporadic human cancers examined $(18,36,39)$. However, its role in intracranial meningiomas has not been satiable explained.

In the present study we have demonstrated the constant and relatively high frequency of $T P 53$ 's mutations in its exon 4 . It has been reported that the most common TP53 mutations are missense mutations representing $80 \%$ of all mutations reported and this was also confirmed with our study. Most missense TP53 mutations disrupt p53 DNA binding. The strong selection for TP53 missense mutations can be explained with the production of mutant p53 proteins. It has been shown that such mutant proteins have lost wild-type p53 tumor suppression functions and acquired the oncogenic gain-of-function that enables them to inactivate other p53 family members, for instance tumor proteins p63 and p73 (40) and promote tumor progression and metastasis. It is also important to take into account that a fraction of TP53 mutations represent frameshift mutations that ultimately lead to nonsense that will produce truncated p53 proteins with impaired wild-type function (18).

Mutation analysis of exon 4 of TP53 gene that we conducted provided valuable information on $\mathrm{p} 53$ protein behavior in intracranial meningioma. Our investigation demonstrates that mutations that impact the p53's transactivation domain and DNA binding domain are present in our group of tumors, which represents novel result for meningioma and further confirmation on the importance of this gene region. DNA binding domain (DBD, residues 102-292) is an important domain by which the protein binds to DNA is located in the central part of the protein and is partially coded by exon 4 . Nevertheless, exon 4 also codes for the transactivation domain (AD2, residues 43-92) of p53 protein, in which functionally important proline rich segments lie (PRD, residues 64-92) $(5,41)$. The reason for choosing to study this exon is primarily because it shows high frequency of mutations in other cancer types, and because it is the only TP53's exon that partially codes for 3 different functionally important domains of p53 protein and also possesses an internal promoter (41). Furthermore, Verheijen et al (42) indicated the involvement of exon 4 in meningioma since different migration patterns were evidenced in 10 out of 17 investigated cases by PCR-SSCP. We found nucleotide alterations in $60.42 \%$ of investigated meningiomas. This relatively high frequency was primarily distributed to codons 72 and 62 . A total of $32.5 \%$ of meningioma investigated by our study showed nucleotide alterations in codons 72 and 62 (each making $44.8 \%$ of total mutations found) when compared to blood DNA and database sequences (IARC T53 Database http://p53.iarc.fr/TP53GeneVariations. aspx; p53 Knowledge base http://p53.bii.a-star.edu. sg/index.php), making these changes highly represented in meningioma. The codon 72 is a known single nucleotide polymorphism reviewed by expert panel and validated, which results in Arg72Pro amino acid change (c.215G>C; p.R72P; rs1042522) and frequently differs in tumors (43). The codon is coding within the polyproline domain of $\mathrm{p} 53$ protein well known for harboring many tumor-associated mutations. The resulting structural variant of the protein differs in molecular functions since it has been shown that this proline-rich region is important for p53's apoptotic role and the Arg variant has stronger ability to induce apoptosis. Also, it has been proposed that the Arg variant binds and inactivates p73 more efficiently thus improving p53's suppressive functions. Of note is that we have found $62 \%$ meningioma with Pro variant and 38\% with Arg variant. Many studies indicate that this polymorphism could be linked to cancer susceptibility to lung, breast, colon and gastric cancers and this change was recently reevaluated and classified as missense and potentially linked to disease by IARC database. Its differences in ethnicity and response to chemotherapeutic treatments has also been documented $(44,45)$. Chang et al $(16)$ also report on the variability of the codon 72 found among meningioma cases with allelic frequencies of 45.6\% (Arg72/Arg72), 44.6\% (Arg72/Pro72), and $9.8 \%$ (Pro72/Pro72).

The deletion of nucleotide A at codon 62 (c.186A $>$ del; p.E62) that we observed in $27.1 \%$ of patients, and which represented $44.8 \%$ of total mutational burden, could introduce a potential frameshift and truncation of the protein in meningioma patients. We confirmed this finding at the protein level where the majority of patients with this deletion lacked p53 protein expression. Other nucleotide substitutions were found on codon 105 in $10.42 \%$ of meningiomas (c. $315 \mathrm{C}>\mathrm{T}$; silent mutation); $8.3 \%$ insertions on codon 70 (c.209_210insG) introducing frameshift; $6.25 \%$ on codons 64 (c.190C $>\mathrm{G}$; missense); 82 (245C $>$ T; 245delC; and 243_244insA) and 104 (c.312G $>$ A) each; $4.2 \%$ on codons 108 (c.322G $>$ C), 71 (c.213C $>\mathrm{A}), 73$ (c.217G >A;), 91 (c.271T>C) and 100 (c.300G $>$ T) each. Codons that harbored only one nucleotide change $(2.1 \%)$ were codons 68 (c.202_203insT), 77 (c.229C >T), 88 (c.263C $>$ G) and 92 (c.276C $>$ A). Although when we compared our findings with the different databases: IARC TP53 mutation database, (http://p53.iarc.fr/TP53GeneVariations.aspx), Ensamble (https://www.ensembl.org/Homo_sapiens/Gene/Sequence $? \mathrm{db}=$ core; $\mathrm{g}=\mathrm{ENSG} 00000141510 ; \mathrm{r}=17: 7661779-7687550)$ and COSMIC (https://cancer.sanger.ac.uk/cosmic) we observed that the alterations were already reported but (except for the codon 72) not for meningiomas. Altogether we have found $46.6 \%$ of missense alterations, $34.5 \%$ of frameshift and $19 \%$ of silent mutations. Variants novel for meningioma we reported to Leiden Open Variation Database (LOVD).

The evaluation of pathogenicity and protein function showed that the majority of alterations were not validated. However, further investigation on functional predictions showed that seven alterations (in codons 82, 91, 108, 104, 105, 70 and 92) were characterized as deleterious and possibly damaging by PolyPhen-2 and Mutation Taster2 tools amounting to $44.4 \%$ of total mutations. Predicted benign alterations also numbered $44.4 \%$. There were $24.1 \%$ mutations whose transcriptional activity was evaluated as functional or partially functional.

Since mutations of TP53 (17q) have been reported to be rare in meningiomas, a general view emerged that other molecular regulators of the $\mathrm{p} 53$ pathway are the ones mutationally targeted and responsible $(20,46)$. Some authors 
propose that p53 aberrations in meningiomas are probably related to mechanisms controlling p53 rather than affecting the gene itself (47). Thus most probable candidates being tumor suppressors CDKN2A/p16INKa (encoding p16), p14ARF (encoding p14), and CDKN2B/p15ARF (encoding p15). p14 is involved with regulating cell apoptosis through modulation of the p53 pathway, and p16 and p15 control cell cycle progress through the G1/S-phase checkpoint (48). However, there are opposing papers reporting on constantly found point mutations $(9,13,49)$ and COSMIC reports on three missense substitutions in TP53 in meningioma. Cho et al (12) investigated exons 5 to 8 of the TP53 gene by SSCP and found 7 mutations (38.9\%). Two meningioma cases had mutations on exon 5 , three on exon 7 and two on exon 8 . Based on the present sudy we believe that the number of cases studied specifically for TP53 mutations across literature is rather low targeting only several exons and therefore account for such conclusions.

Several studies collectively showed that p53 positivity is correlated with the meningioma grade $(12,13,24,25,47,50-52)$. Positive p53 protein expression was found in $77 \%$ of grade III meningiomas (53) and meningiomas with higher malignancy grades displayed higher frequency of nuclear p53 positivity (26). Similarly, studies by Amatya et al (15) and Csonka et al (54) found a higher staining intensity in the high-grade tumors which suggested that the p53 activation might be associated to tumor progression. Our own previous analyses showed that p53 positivity was significantly associated with higher meningioma grades. We speculated that the positive nuclear signal was indicative of mutant p53 proteins because it has been shown that they are more stable and generally highly expressed in cancer (6). However, present mutational analysis demonstrated that the group of samples harboring mutations expressed significantly less protein $(\mathrm{P}=0.04$; Fig. 2D) from the group without mutations, indicating that mutational gain-of-function could not be attributed to meningioma, but rather the classical behavior of tumour suppressor loss. In the present study codons 72 and 62 were most frequently changed in both meningothelial and transitional subtypes, while codon 105 in the transitional subtype, but the observed frequencies were not statistically significantly associated to meningioma histopathology, nor to their grades, probably because of small number of meningiomas with grades II and III.

Our previous results clearly demonstrated that the inactivation of tumor suppressor gene TP53 in meningioma was not achieved through allelic loss (55). Although usual mode of action for tumor suppressor genes is the loss of heterozygosity followed by mutations, action mechanisms should not be generalized for all suppressor genes and all tumors $(38,56)$.

The p53's fundamental tumor suppressive role, as well as its oncogenic mechanisms are still incompletely explained. Mutations of TP53 and the consequent heterotetramerization of wild-type and mutant p53 often stabilize the complex leading to abrogation of normal p53 function in a cell with both mutant and wild type alleles $(12,24,25,47,50,51)$. Moreover, isoforms of p53 family have been found in human arising through alternative splicing, use of alternative translation site or alternative promoter $(19,57)$.
The prevailing base substitutions of TP53 and the resultant inactivation of p53 allow evasion of apoptosis and rapid carcinogenesis. Nevertheless, occurring variants may be substrate for specific cancer risk.

Sex has been known to be an intrinsic risk factor for meningeoma. The twice as high incidence observed in women as compared to men is related to female sex hormones especially during the reproductive life. We have found that women exhibited significantly higher frequency of total nucleotide alterations when compared to males (Chi square $=3.802$; sig=0.049). The analysis of specific codon changes demonstrated that females had significantly more alterations of codon 62 than male patients (Chi square $=10.447$; sig=0.001). Although it has been demonstrated that the protective function of the p53 protein declines with age (58), our results could not establish such a connection, TP53 nucleotide alterations were not significantly associated to age.

Although highly mutated in cancer, the spectrum and the frequency of TP53 mutations vary between tumor types. There are also views that each tumor-associated TP53 missense amino acid change will have a unique effect on p53 structure, its binding to the DNA and its other interactions. Thus, mutant p53 proteins can be regarded as a heterogeneous group of proteins with various degrees of normal tumor suppressor function loss and various degrees of gain of oncogenic properties (18).

In conclusion, the present study shows that nucleotide alterations found in TP53's exon 4 are much more frequent than previously reported. This exon partially codes for 3 functionally important domains of p53 protein and highly represented changed codons could be responsible but need to be functionally validated. Our results contribute to meningioma genetic profile.

\section{Acknowledgements}

Not applicable.

\section{Funding}

The present study was funded by the Croatian Science Foundation (grant no. 6625) and the Scientific Centre of Excellence for Basic, Clinical and Translational Neuroscience (project 'Experimental and clinical research of hypoxic-ischemic damage in perinatal and adult brain'; grant no. GA KK01.1.1.01.0007; funded by the European Union through the European Regional Development Fund).

\section{Availability of data and materials}

The datasets used and/or analyzed during the present study are available from the corresponding author on reasonable request.

\section{Authors' contributions}

$\mathrm{AB}$ and NPŠ designed the current study. AB, AK, RH, TV and NPŠ acquired the data. AB, TV and NPŠ analyzed the data. AB, RH, TV and NPŠ interpreted the data. AB and AK performed the experiments. AB, AK, RH and NPŠ wrote the manuscript. AB, AK, RH, TV and NPŠ revised the manuscript 
for intellectual content. All authors read and approved the final manuscript.

\section{Ethics approval and consent to participate}

All procedures of the current study involving human participants were performed in accordance with the ethical standards of the Institutional and National Research Committee and with the Declaration of Helsinki. Ethical approval was received from the Ethical Committee of the School of Medicine, University Zagreb (approval no. 380-59-10106-14-55/147; Class, 641-01/14-02/01,1.07. 2014) and the University Hospital Center 'Sisters of Charity' (approval no. EP-7426/14-9,11. 06. 2014). Informed consent was obtained from all individual participants included in the study.

\section{Patient consent for publication}

Not applicable.

\section{Competing interests}

The authors declare that they have no competing interests.

\section{References}

1. Domingues P, González-Tablas M, Otero Á, Pascual D, Ruiz L, Miranda D, Sousa P, Gonçalves JM, Lopes MC, Orfao A and Tabernero MD: Genetic/molecular alterations of meningiomas and the signaling pathways targeted. Oncotarget 6: 10671-10688, 2015.

2. Pećina-Šlaus N, Nikuseva Martic T, Tomas D, Beros V, Zeljko M and Cupic H: Meningiomas exhibit loss of heterozygosity of the APC gene. J Neurooncol 87: 63-70, 2008

3. El-Habr EA, Levidou G, Trigka EA, Sakalidou J, Piperi C, Chatziandreou I, Spyropoulou A, Soldatos R, Tomara G, Petraki K, et al: Complex interactions between the components of the PI3K/AKT/mTOR pathway, and with components of MAPK, JAK/STAT and Notch-1 pathways, indicate their involvement in meningioma development. Virchows Arch 465: 473-485, 2014.

4. Al-Khalaf HH, Lach B, Allam A, Lakhani A, Alrokayan SA and Aboussekhra A: The p53/p21 DNA damage-signaling pathway is defective in most meningioma cells. J Neurooncol 83: 9-15, 2007.

5. Sorrell AD, Espenschied CR, Culver JO and Weitzel JN: Tumor protein p53 (TP53) testing and Li-Fraumeni syndrome: Current status of clinical applications and future directions. Mol Diagn Ther 17: 31-47, 2013.

6. Muller PA and Vousden KH: Mutant $\mathrm{p} 53$ in cancer: New functions and therapeutic opportunities. Cancer Cell 25: 304-317, 2014.

7. Ohgaki H, Eibl RH, Schwab M, Reichel MB, Mariani L, Gehring M, Petersen I, Höll T, Wiestler OD and Kleihues P: Mutations of the p53 tumor suppressor gene in neoplasms of the human nervous system. Mol Carcinog 8: 74-80, 1993.

8. Ellison DW, Lunec J, Gallagher PJ, Steart PV, Jaros E and Gatter KC: Accumulation of wild-type p53 in meningiomas. Neuropathol Appl Neurobiol 21: 136-142, 1995.

9. Joachim T, Ram Z, Rappaport ZH, Simon M, Schramm J, Wiestler OD and von Deimling A: Comparative analysis of the NF2, TP53, PTEN, KRAS, NRAS and HRAS genes in sporadic and radiation-induced human meningiomas. Int J Cancer 94: 218-221, 2001

10. Das A, Tan WL and Smith DR: p53 point mutation is rare in meningiomas from Singaporean patients. Asian J Surg 28: 7-10, 2005.

11. Pykett MJ, Landers J and George DL: Expression patterns of the p53 tumor suppressor gene and the $\mathrm{mdm} 2$ proto-oncogene in human meningiomas. J Neurooncol 32: 39-44,1997.

12. Cho H, Ha SY, Park SH, Park K and Chae YS: Role of p53 gene mutation in tumor aggressiveness of intracranial meningiomas. J Korean Med Sci 14: 199-205, 1999.
13. Wang JL, Zhang ZJ, Hartman M, Smits A, Westermark B, Muhr C and Nistér M: Detection of TP53 gene mutation in human meningiomas: A study using immunohistochemistry, polymerase chain reaction/single-strand conformation polymorphism and DNA sequencing techniques on paraffin-embedded samples. Int J Cancer 64: 223-228, 1995.

14. Ohkoudo M, Sawa H, Hara M, Saruta K, Aiso T, Ohki R, Yamamoto H, Maemura E, Shiina Y, Fujii M and Saito I: Expression of p53, MDM2 protein and Ki-67 antigen in recurrent meningiomas. J Neurooncol 38: 41-49, 1998.

15. Amatya VJ, Takeshima Y and Inai K: Methylation of p14(ARF) gene in meningiomas and its correlation to the p53 expression and mutation. Mod Pathol 17: 705-710, 2004.

16. Chang Z, Guo CL, Ahronowitz I, Stemmer-Rachamimov AO, MacCollin M and Nunes FP: A role for the p53 pathway in the pathology of meningiomas with NF2 loss. J Neurooncol 91: 265-270, 2009.

17. Zhang X, Gejman R, Mahta A, Zhong Y, Rice KA, Zhou Y, Cheunsuchon P, Louis DN and Klibanski A: Maternally expressed gene 3 , an imprinted noncoding RNA gene, is associated with meningioma pathogenesis and progression. Cancer Res 70: 2350-2358, 2010.

18. Bykov VJN, Eriksson SE, Bianchi J and Wiman KG: Targeting mutant p53 for efficient cancer therapy. Nat Rev Cancer 18: 89-102, 2018.

19. Marcel V, Nguyen Van Long F and Diaz JJ: 40 years of research put p53 in translation. Cancers (Basel) 10: E152, 2018.

20. Riemenschneider MJ, Perry A and Reifenberger G: Histological classification and molecular genetics of meningiomas. Lancet Neurol 5: 1045-1054, 2006.

21. Louis DN, Perry A, Reifenberger G, von Deimling A, Figarella-Branger D, Cavenee WK, Ohgaki H, Wiestler OD, Kleihues P and Ellison DW: The 2016 World Health Organization classification of tumors of the central nervous system: A summary. Acta Neuropathol 131: 803-820, 2016.

22. Fathi AR and Roelcke U: Meningioma. Curr Neurol Neurosci Rep 13: 337, 2013.

23. He S, Pham MH, Pease M, Zada G, Giannotta SL, Wang K and Mack WJ: A review of epigenetic and gene expression alterations associated with intracranial meningiomas. Neurosurg Focus 35: E5, 2013.

24. Pavelin S, Becic K, Forempoher G, Mrklic I, Pogorelic Z, Titlic $\mathrm{M}$ and Andelinovic S: Expression of Ki-67 and p53 in meningiomas. Neoplasma 60: 480-485, 2013.

25. Narla S, Uppin MS, Saradhi MV, Sahu BP, Purohit AK and Sundaram C: Assessment of expression of epidermal growth factor receptor and p53 in meningiomas. Neurol India 62: 37-41, 2014.

26. Trott G, Pereira-Lima JF, Leães CG, Ferreira NP, BarbosaCoutinho LM and Oliveira MC: Abundant immunohistochemical expression of dopamine D2 receptor and p53 protein in meningiomas: Follow-up, relation to gender, age, tumor grade, and recurrence. Braz J Med Biol Res 48: 415-419, 2015.

27. Preusser M, Brastianos PK and Mawrin C: Advances in meningioma genetics: Novel therapeutic opportunities. Nat Rev Neurol 14: 106-115, 2018.

28. Wang $\mathrm{N}$ and Osswald M: Meningiomas: Overview and new directions in therapy. Semin Neurol 38: 112-120, 2018.

29. Pereira BJA, Oba-Shinjo SM, de Almeida AN and Marie SKN: Molecular alterations in meningiomas: Literature review. Clin Neurol Neurosurg 176: 89-96, 2019.

30. Pećina-Šlaus N: Merlin the NF2 gene product. Pathol Oncol Res 19: 365-373, 2013.

31. Pećina-Šlaus N, Kafka A and Lechpammer M: Molecular genetics of intracranial meningiomas with emphasis on canonical Wnt signalling. Cancers (Basel) 8: E67, 2016.

32. Adzhubei IA, Schmidt S, Peshkin L, Ramensky VE, Gerasimova A, Bork P, Kondrashov AS and Sunyaev SR: A method and server for predicting damaging missense mutations. Nat Methods 7: 248-249, 2010.

33. Sim NL, Kumar P, Hu J, Henikoff S, Schneider G and Ng PC: SIFT web server: Predicting effects of amino acid substitutions on proteins. Nucleic Acids Res 40 (Web Server Issue): W452-W457, 2012.

34. Schwarz JM, Cooper DN, Schuelke M and Seelow D: MutationTaster2: Mutation prediction for the deep-sequencing age. Nat Methods 11: 361-362, 2014.

35. Olivier M, Langerød A, Carrieri P, Bergh J, Klaar S, Eyfjord J, Theillet $\mathrm{C}$, Rodriguez $\mathrm{C}$, Lidereau R, Bièche $\mathrm{I}$, et al: The clinical value of somatic TP53 gene mutations in 1,794 patients with breast cancer. Clin Cancer Res 12: 1157-1167, 2006. 
36. Lindenbergh-van der Plas M, Brakenhoff RH, Kuik DJ, Buijze M, Bloemena E, Snijders PJ, Leemans CR and Braakhuis BJ: Prognostic significance of truncating TP53 mutations in head and neck squamous cell carcinoma. Clin Cancer Res 17: 3733-3741, 2011.

37. Omura G, Ando M, Ebihara Y, Saito Y, Kobayashi K, Fukuoka O, Akashi K, Yoshida M, Asakage T and Yamasoba T: The prognostic value of TP53 mutations in hypopharyngeal squamous cell carcinoma. BMC Cancer 17: 898, 2017.

38. Bouaoun L, Sonkin D, Ardin M, Hollstein M, Byrnes G Zavadil J and Olivier M: TP53 variations in human cancers: New Lessons from the IARC TP53 Database and Genomics Data. Hum Mutat 37: 865-876, 2016.

39. Bieging KT, Mello SS and Attardi LD: Unravelling mechanisms of p53-mediated tumour suppression. Nat Rev Cancer 14: 359-730, 2014

40. Walerych D, Lisek K and Del Sal G: Mutant p53: One, no one, and one hundred thousand. Front Oncol 5: 289, 2015.

41. Harms KL and Chen X: The functional domains in p53 family proteins exhibit both common and distinct properties. Cell Death Differ 13: 890-897, 2006.

42. Verheijen FM, Sprong M, Kloosterman JM, Blaauw G, Thijssen JH and Blankenstein MA: TP53 mutations in human meningiomas. Int J Biol Markers 17: 42-48, 2002.

43. Faria MH, Neves Filho EH, Alves MK, Burbano RM, de Moraes Filho MO and Rabenhorst SH: TP53 mutations in astrocytic gliomas: An association with histological grade, TP53 codon 72 polymorphism and p53 expression. APMIS 120: 882-889, 2012.

44. Grochola LF, Zeron-Medina J, Mériaux S and Bond GL: Single-nucleotide polymorphisms in the p53 signaling pathway. Cold Spring Harb Perspect Biol 2: a001032, 2010

45. Naccarati A, Polakova V, Pardini B, Vodickova L, Hemminki K, Kumar R and Vodicka P: Mutations and polymorphisms in TP53 gene-an overview on the role in colorectal cancer. Mutagenesis 27: 211-218, 2012.

46. Choy W, Kim W, Nagasawa D, Stramotas S, Yew A, Gopen Q, Parsa AT and Yang I: The molecular genetics and tumor pathogenesis of meningiomas and the future directions of meningioma treatments. Neurosurg Focus 30: E6, 2011.

47. Terzi A, Saglam EA, Barak A and Soylemezoglu F: The significance of immunohistochemical expression of Ki-67, p53, p21, and p16 in meningiomas tissue arrays. Pathol Res Pract 204: 305-314, 2008

48. Boström J, Meyer-Puttlitz B, Wolter M, Blaschke B, Weber RG, Lichter P, Ichimura K, Collins VP and Reifenberger G: Alterations of the tumor suppressor genes CDKN2A (p16(INK4a)), p14(ARF), CDKN2B (p15(INK4b)), and CDKN2C (p18(INK4c)) in atypica and anaplastic meningiomas. Am J Pathol 159: 661-669, 2001.
49. Mashiyama S, Murakami Y, Yoshimoto T, Sekiya T and Hayashi K: Detection of p53 gene mutations in human brain tumors by single-strand conformation polymorphism analysis of polymerase chain reaction products. Oncogene 6: 1313-1318, 1991.

50. Matsuno A, Nagashima T, Matsuura R, Tanaka H, Hirakawa M, Murakami M, Tamura A and Kirino T: Correlation between MIB-1 staining index and the immunoreactivity of p53 protein in recurrent and non-recurrent meningiomas. Am J Clin Pathol 106: 776-781, 1996.

51. Nagashima G, Aoyagi M, Yamamoto M, Yamamoto S, Wakimoto H, Ohno K, Yamamoto K and Hirakawa K: P53 overexpression and proliferative potential in malignant meningiomas. Acta Neurochir (Wien) 141: 53-61, 1999.

52. Ozen O, Demirhan B and Altinörs N: Correlation between histological grade and MIB-1 and p53 immunoreactivity in meningiomas. Clin Neuropathol 24: 219-224, 2005.

53. Karamitopoulou E, Perentes E, Tolnay $M$ and Probst A: Prognostic significance of MIB-1, p53, and bcl-2 immunoreactivity in meningiomas. Hum Pathol 29: 140-145, 1998.

54. Csonka T, Murnyák B, Szepesi R, Kurucz A, Klekner Á and Hortobágyi T: Poly(ADP-ribose) polymerase-1 (PARP1) and p53 labelling index correlates with tumour grade in meningiomas. Folia Neuropathol 52: 111-120, 2014

55. Pećina-Šlaus N, Kafka A, Vladušić T, Tomas D, Logara M, Skoko $\mathrm{J}$ and Hrašćan R: Loss of p53 expression is accompanied with upregulation of beta-catenin in meningiomas: A concomitant reciprocal expression. Int J Exp Pathol 97: 159-169, 2016.

56. Leroy B, Fournier JL, Ishioka C, Monti P, Inga A, Fronza G and Soussi T: The TP53 website: An integrative resource centre for the TP53 mutation database and TP53 mutant analysis. Nucleic Acids Res 41 (Database Issue): D962-D969, 2013.

57. Marcel V and Hainaut P: p53 isoforms-a conspiracy to kidnap p53 tumor suppressor activity? Cell Mol Life Sci 66: 391-406, 2009.

58. Feng Z, Hu W, Teresky AK, Hernando E, Cordon-Cardo C and Levine AJ: Declining p53 function in the aging process: A possible mechanism for the increased tumor incidence in older populations. Proc Natl Acad Sci USA 104: 16633-16638, 2007.

(i) $\odot$ This work is licensed under a Creative Commons Attribution-NonCommercial-NoDerivatives 4.0 International (CC BY-NC-ND 4.0) License. 